REVISTA DE LA FACULTAD

DE CIENCIAS DE LA SALUD

\title{
Implementación y sostenibilidad de las guías de enfermería basadas en la evidencia: modelo de la RNAO
}

Implementations and sustainability of evidence-based nursing guides: RNAO model

Implementação e sustentabilidade de guias de enfermagem baseados em evidências: modelo RNAO

Lina María Granados-Oliveros, Enf. ${ }^{1}$ (D), Maribel Esparza-Bohorquez, Enf. ${ }^{2}$ (D)

1. Enfermera, Coordinadora de Hospitalización, Fundación Oftalmológica de Santander (FOSCAL), Floridablanca, Santander, Colombia.

2. Enfermera, Jefe División de Enfermería, Fundación Oftalmológica de Santander (FOSCAL), Floridablanca, Santander, Colombia.

Correspondencia. Lina María Granados Oliveros, Coordinadora de Hospitalización, Fundación Oftalmológica de Santander (FOSCAL), Torre Milton Salazar, Piso 3, Urbanización el Bosque. Floridablanca, Santander, Colombia. Email. lina.granados@foscal.com.co

Cómo citar: Granados-Oliveros LM, Esparza-Bohorquez M. Implementación y sostenibilidad de las guías de enfermería basadas en la evidencia: modelo de la RNAO. MedUNAB. 2020;23(1):85-94. doi:10.29375/01237047.3725

\section{INFORMACIÓN DEL ARTÍCULO:}

Artículo recibido: 23 de agosto de 2019

Artículo aceptado: 16 de enero de 2020

Doi: https://doi.org/10.29375/01237047.3725

\section{RESUMEN}

Introducción. Se reconoce que uno de los aportes de la enfermería basada en la evidencia (EBE) es el mejoramiento de la calidad de la atención y existen organizaciones como la Asociación de Enfermeras Registradas de Ontario (RNAO) que han desarrollado guías de práctica clínica y estrategias metodológicas para su implementación. El objetivo de este artículo es describir la experiencia y reflexiones sobre el proceso de implementación de guías de EBE de la RNAO, haciendo énfasis en los resultados de adherencia y sostenibilidad de tres guías en una institución de tercer nivel. Temas de reflexión. La organización RNAO ha elaborado una 
herramienta de implementación según el modelo de Proceso de Conocimiento para la Acción que implica seis fases: identificación del problema, adaptación al contexto local, evaluación de los facilitadores y barreras para el uso del conocimiento, adaptación, implantación de intervenciones / estrategias de implementación, monitorización, evaluación y sostenibilidad. Todas estas se adelantaron para implementar inicialmente tres guías ("Valoración y manejo del dolor", "Prevención de caídas y lesiones derivadas de las caídas" y "Valoración del riesgo de lesiones de piel por presión"), en una institución de tercer nivel. Conclusiones. La auditoría y verificación periódica de las estrategias implementadas promueve la adherencia del personal de los servicios en el cumplimiento de las metas institucionales y la sostenibilidad del cambio logrado.

Palabras claves:

Enfermería; Enfermería Basada en la Evidencia; Práctica Clínica Basada en la Evidencia; Calidad de la Atención en Salud; Manejo del dolor; Accidentes por Caídas; Cuidados de la Piel.

\section{ABSTRACT}

Introduction. It is recognized that the evidence-based nursing (EBN) contributed to enhancing the quality of care and that there are organizations such as the Registered Nurses' Association of Ontario (RNAO) that have developed guides on clinical practice and methodological strategies for their implementation. This article describes the experience and reflections on the implementation process of RNAO's EBN guides, focusing on the sustainability and adhesion results of three guides implemented in a tertiary institution. Reflection topics. RNAO has created an implementation tool according to the knowledge-to-action process model which consists of six phases: problem identification, local context adaptation, assessment of facilitating agents and of obstacles for the use of knowledge, adjustment, deployment of interventions / implementation strategies, monitoring, evaluation and sustainability. All these phases were advanced to initially implement three guides ("Assessing and managing pain", "Preventing falls and injuries from falls" and "Assessing skin lesions risk due to pressure") at a tertiary institution. Conclusions. The periodic audit and verification of the strategies implemented promotes compliance by the service personnel as regards institutional goals and the sustainability of the change achieved

Keywords:

Nursing; Evidence-Based nursing; Evidence-Based Practice; Quality of Health Care; Pain Management; Accidental Falls; Skin Care.

\section{RESUMO}

Introdução. Reconhece-se que uma das contribuições da enfermagem baseada em evidências (EBE) é a melhoria da qualidade do atendimento, e existem organizações como a Associação de Enfermeiras Registradas de Ontário (RNAO) que tem desenvolvido diretrizes de prática clínica e estratégias metodológicas para sua implementação. Objetivo. Descrever a experiência e as reflexões sobre o processo de implementação dos guias de EBE do RNAO, enfatizando os resultados da adesão e sustentabilidade de três guias em uma instituição de terceiro nível. Refleção. A organização RNAO desenvolveu uma ferramenta de implementação de acordo com o modelo Processo de Conhecimento para a Ação que envolve seis fases: identificação do problema, adaptação ao contexto local, avaliação de facilitadores e limitações ao uso do conhecimento, adaptação, implementação de intervenções/estratégias de implementação, monitoramento, avaliação e sustentabilidade. Todas estas foram organizadas para implementar inicialmente três guias ("Avaliação e tratamento da dor", "Prevenção de quedas e lesões resultantes de quedas" e "Avaliação do risco de lesões na pele devido à pressão"), em uma instituição de terceiro nível. Conclusões. A auditoria e a verificação periódica das estratégias implementadas promovem a adesão do pessoal de serviço no cumprimento dos objetivos institucionais e a sustentabilidade da mudança alcançada.

Palavras-chave:

Enfermagem; Enfermagem Baseada em Evidências; Prática Clínica Baseada em Evidências; Qualidade da Assistência à Saúde; Manejo da Dor; Acidentes por Quedas; Higiene da Pele. 


\section{Introducción}

La Práctica Basada en la Evidencia (PBE) tiene su El mejoramiento de la calidad de la atención puede lograrse por medio de la implementación de prácticas de enfermería basada en la evidencia (EBE). La enfermería basada en la evidencia es un movimiento nuevo que se apoya en las bases conceptuales de la medicina basada en la evidencia. Según la definición del profesor Sackett et al., la medicina basada en la evidencia es la utilización consciente, explícita y juiciosa de la mejor evidencia clínica disponible para tomar decisiones sobre el cuidado de cada paciente (1). Está basada en cuatro conceptos generales: la investigación, la experiencia del clínico, las preferencias/valores de los usuarios y los recursos disponibles (2).

La enfermería basada en la evidencia (EBE) se desarrolló inicialmente en países de habla inglesa. En 1977, se realizaron en Reino Unido las primeras conferencias nacionales e internacionales de EBE (3). Desde la perspectiva de la evidencia, la concepción de la práctica de la enfermería se ve modificada, en la medida en que brinda la posibilidad de revisar, reconstruir y rehacer los servicios que presta la enfermera a la luz de pruebas válidas (aproximación a la verdad) y útiles (aplicabilidad clínica) procedentes de la investigación. Los cuidados o práctica basada en la evidencia aplicada en una disciplina aumentan la calidad de los cuidados, la seguridad en los pacientes y el manejo de los riesgos en la práctica (4). En América y a nivel mundial, se destaca la labor realizada por la Asociación de Enfermeras Registradas de Ontario (RNAO) desde 1999 (5), que ha producido una amplia serie de guías de EBE, en los ámbitos asistenciales, académicos y administrativos.

En el presente artículo se describe la experiencia en la Fundación Ofalmológica de Santander - Clínica Ardila Lülle FOSCAL (Institución de Salud de tercer nivel de atención en Colombia) del proceso de implementación de guías de EBE de la RNAO, haciendo énfasis en los resultados de adherencia y sostenibilidad. La enfermera debe promover un liderazgo efectivo en la gestión de los equipos de trabajo de su dependencia, para ello debe proponer programas de evaluación y mejoramiento continúo del cuidado de enfermería (6). La incorporación de las recomendaciones de las guías a la práctica en cada una de las áreas y en la cultura organizacional, permite lograr los cambios de la práctica por el personal en el proceso de atención; además, se logra disminuir la variabilidad de la práctica clínica, al dar respuesta a las necesidades del cuidado durante el proceso asistencial (7). La incorporación de los resultados a la práctica clínica ha sido posible gracias a la implementación de guías de enfermería basadas en la evidencia (EBE), de tal manera que se han creado instituciones dedicadas especialmente en el trabajo o enfoque de producción de estas (8). Un modelo de esto lo constituye la Asociación de Enfermeras Registradas de Ontario (RNAO).

La RNAO ha trabajado desde hace más de 12 años en esta iniciativa, desarrolló el Programa de Directrices de las Mejores Prácticas de Enfermería (BPG), la cual cuenta con una herramienta rigurosa para el proceso de elaboración de las guías, que incluye: la identificación del tema específico de la BPG, una revisión sistemática de la mejor y más actualizada evidencia disponible, la selección de un panel de expertos (enfermeros, otras profesiones, pacientes y comunidad) que desarrollen las recomendaciones clínicas, organizacionales, educativas y de sistema derivadas de la evidencia, y la publicación final de la directriz o guía (9).

La RNAO cuenta con 54 guías de buenas prácticas de las cuales 8 son fundacionales, 24 de gestión clínica, 9 de sistema y ambiente de trabajo saludable y 13 de salud de la población y salud pública (10). Actualmente las guías de EBE son ampliamente utilizadas por más de 60 instituciones a nivel mundial reconocidas como Best Practice Spotlight Organizations (BPSO) o Institución Comprometida con la Excelencia (11). En América Latina, la guía de EBE de la RNAO se utiliza actualmente en Chile en diversas instituciones a nivel asistencial como la clínica Las Condes, y a nivel académico, en la Universidad de Chile.

En Colombia, las guías de la RNAO son referente en dos instituciones de salud: la Fundación Cardioinfantil y Fundación Oftalmológica de Santander Clínica Carlos Ardila Lulle (FOSCAL), y a nivel académico en la Universidad Autónoma de Bucaramanga (UNAB). Con el presente artículo se quiere describir la experiencia y reflexiones sobre el proceso de implementación de guías de EBE de la RNAO, haciendo énfasis en los resultados de adherencia y sostenibilidad de tres guías en una institución de tercer nivel.

Para lograr lo anterior, la RNAO elaboró la herramienta de implementación según el modelo de Proceso de Conocimiento para la Acción (S. Straus, J. Tetroe, I. Graham), esencial para la trasmisión del conocimiento (8). La implementación de las EBE implicó seguir la metodología propuesta que contempló las siguientes fases:

Primera fase: Identificación del problema: se identificaron las necesidades de la institución y se 
examinaron las posibles guías de buenas prácticas que podían ayudar a resolver el problema.

Segunda fase: Adaptación al contexto local: se identificó y analizó el contexto. Se consideró como una de las etapas principales del proceso de implementación, dado que el entorno afectaba la práctica y constituía un mediador importante en la difusión de cambios. En esta fase se contemplaron 3 partes:

* Parte A: Desarrollo de una infraestructura para la implantación de las mejores prácticas.

* Parte B: Identificación de las partes interesadas, análisis y compromiso para liderar el proceso de implantación.

* Parte C: Evaluación del entorno y los recursos necesarios en la gestión y monitorización de la implantación de las Guías de Buenas Prácticas (GBP).

Tercera fase: Evaluación de los facilitadores y barreras para el uso del conocimiento: se identificaron las barreras que impiden, parcial o totalmente, la implementación, como la falta de conocimiento, las actitudes y la resistencia al cambio. Se evaluó cómo intervenirlas con elementos que facilitan la transferencia de conocimiento. Se evaluó el entorno y los colaboradores relevantes.

Cuarta fase: Adaptación, implantación de intervenciones / Estrategias de implantación: según la experiencia de la RNAO, es difícil cambiar el comportamiento de los profesionales de enfermería. Es por esta razón que las estrategias de implantación deben ser cuidadosamente planeadas y utilizadas en forma conjunta. Dentro de las estrategias de implantación se resalta la formación del personal, la cual se realizó a través de talleres, conferencias, trabajos de campo, auditorías, etc. Se debía lograr un feedback oportuno entre el educador y el profesional para que se produjera un cambio en el comportamiento.

En esta fase es importante tener en cuenta el proceso de cambio, con refuerzo en el Modelo de Lewin de Descongelación, Cambio y Recongelación (Figura 1) (8).

En la fase de descongelación, el personal termina entendiendo que el cambio es necesario y se prepara para salir de su zona de confort. En la fase de cambio, el personal está descongelado de sus antiguos hábitos y empieza a avanzar; es necesario dar tiempo para que
Figura 1. Modelo de Lewin de Descongelación, Cambio y Recongelación.

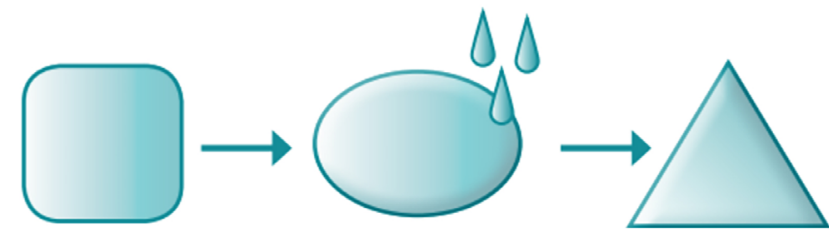

Fuente. Adaptado de Lewin et al. (8)

aprendan sobre el mismo cambio. Y finalmente, la fase de recongelación es el momento donde los cambios se han realizado y se instaura la estabilidad. El personal se acomoda a las nuevas rutinas (9).

Quinta y sexta fase: Motorización, evaluación y sostenibilidad: en esta fase se evalúa el uso y cumplimiento de las recomendaciones, los cambios en el conocimiento y/o las actitudes. Se definieron los indicadores clave para evaluar el proceso de implementación con el fin de monitorear la cultura de la organización y la apropiación del conocimiento. Con la herramienta de RNAO de NQUiRE se realiza el consolidado de esta información al ingresar los datos de los indicadores de cada una de las guías implementadas en cada uno de los servicios (Figura 2). Esto garantiza el monitoreo de la información, permitiendo el seguimiento a la tendencia de los resultados obtenidos con el proceso de incorporación de la evidencia científica a la parte clínica de la institución para trabajar en pro de la sostenibilidad de la atención en los servicios y el monitoreo dinámico con los indicadores establecidos por cada guía, los cuales permiten evidenciar el proceso de cambio y mejora a través de la planificación desde el inicio de la implementación (12).

En el convenio firmado con la RNAO en el año 2012, la FOSCAL se comprometió inicialmente a participar como mínimo durante de tres años, período en el cual se debía cumplir con entregables propuestos para que fuera evaluada la institución y renovado el convenio. Las directrices incluían, entre otras: participar en sesiones regulares de intercambio de conocimiento, monitoreo mediante el reporte de indicadores en la plataforma NQUiRE, planes de mejoramiento y publicaciones. Además, se debía apoyar a otras instituciones aspirantes a BPSO mediante el intercambio de conocimientos y experiencias. Este artículo busca describir la experiencia de la clínica FOSCAL en la implementación de guías de enfermería basadas en la evidencia de la RNAO, con énfasis en el proceso de adherencia y sostenibilidad. 
Figura 2. Herramienta de la RNAO: proceso de conocimiento para la acción.

\section{GBP Herramienta de la RNAO Proceso de Conocimiento para la Acción}

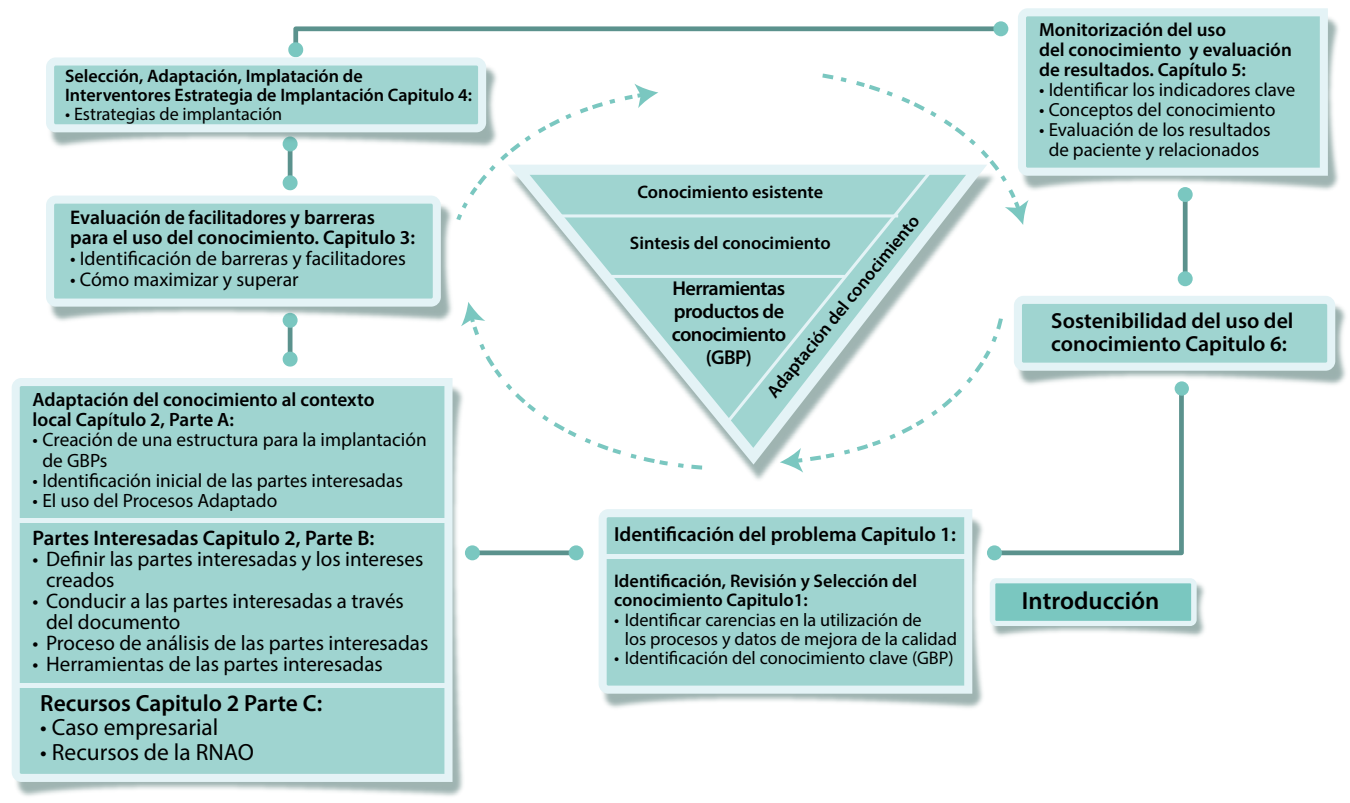

Fuente. Adaptado de Straus S, Tetroe J, Graham I. "Knowledge translation in health care: moving from evidence to practice", 2009.

La división de enfermería de la FOSCAL, institución de tercer nivel de atención, para iniciar el programa de implementación de la EBE, seleccionó las guías de prevención de caídas y lesiones derivadas de las caídas y la guía de valoración del riesgo de úlceras por presión, elegidas por las implicaciones que estos eventos tienen para el usuario, familia e institución. La guía de valoración y manejo del dolor no fue seleccionada, por no contar con un cuidado sistemático, ni intervenciones estandarizadas, para dar calidad y confort al usuario.

\section{Proceso de implementación de las guías EBE en FOSCAL}

La implementación debe ser planeada en relación con el contexto en el que se proporciona el cuidado y en el que influyen factores individuales y organizativos. Uno de estos factores individuales está relacionado con la consideración de la preferencia y los valores del paciente para consensuar sus cuidados. Además de la participación y aceptación de los cuidados por parte del paciente, el juicio crítico y la experiencia del profesional son otros factores individuales sumamente importantes (13). Su naturaleza garantiza el uso racional de las GPC $y$, por lo tanto, establece la diferencia entre la EBE y un libro de recetas de cocina (14).

Teniendo en cuenta la primera fase del proceso de implementación recomendado por la RNAO de guías EBE, la cual se desarrolló desde el año 2013 al 2015 como una primera etapa donde se identificaron los problemas críticos que se podían intervenir a través de esta metodología y así implementar las guías que más impactaban en los problemas identificados, las guías basadas en la evidencia de la RNAO que iniciaron proceso de implementación fueron:

- Guía de valoración y manejo del dolor.

- Prevención de caídas y lesiones derivadas de las caídas.

- Valoración del riesgo de lesiones de piel por presión.

Se evalúo la calidad y viabilidad de la implementación de las guías: con la metodología AGREEII para evaluar el rigor metodológico y transparencia con la cual se elaboran las guías, y con la metodología AGLI para determinar las recomendaciones a implementar. 
Según los resultados descritos en la tabla 1, se seleccionaron las recomendaciones a implementar, mediante la adopción de cada una de las guías a nivel institucional.

Seguidamente, se seleccionaron los servicios mediante la evaluación de la incidencia de eventos relacionados y su impacto. Así, para la guía de piel, los servicios piloto para la aplicación de las recomendaciones de la guía fueron los pisos de medicina interna, el servicio de neurocirugía y la Unidad de Cuidados Intensivos Adultos.

Para la guía de prevención de caídas se seleccionó hospitalización general, y para la guía de valoración y manejo de la guía del dolor, los servicios de sala de partos y de hospitalización de oncología.
Se realizó el proceso de selección y formación de "líderes champions", personas clave en los servicios para la puesta en marcha del proceso. A continuación, se capacitó el personal asistencial y se inició el despliegue del programa con la elaboración de formatos físicos para la recolección de la información en cada servicio. Posteriormente, con adecuaciones y nuevos desarrollos en el sistema de información, se incluyeron los registros en la historia clínica electrónica. En complemento, como parte de la sensibilización al personal, se crearon estrategias para dar a conocer el programa y las guías dentro de la organización: afiches, habladores, mensajes por página web institucional, presentación, folletos, entre otros.

Tabla 1. Resultados de la evaluación metodológica de las guías RNAO seleccionadas para implementación en la Foscal

\begin{tabular}{lcl}
\multicolumn{1}{c}{ Guía } & AGREE II & \multicolumn{1}{c}{ AGLI } \\
\hline Guía de valoración y manejo dolor & $91.3 \%$ & $\begin{array}{l}\text { De 18 recomendaciones, 17 son implementables y una } \\
\text { es implementable con condiciones. }\end{array}$ \\
\hline $\begin{array}{l}\text { Guías de prevención de caídas y } \\
\text { lesiones derivadas de las caídas }\end{array}$ & $89.3 \%$ & $\begin{array}{l}\text { De 13 recomendaciones, 9 son implementables, } \\
\text { implementables con condiciones y 2 no }\end{array}$ \\
\hline $\begin{array}{l}\text { Guía de prevención de úlceras por } \\
\text { presión }\end{array}$ & $81.5 \%$ & $\begin{array}{l}\text { De 31 recomendaciones, 23 son implementables, } 2 \\
\text { implementables con condiciones y 6 no se encuentran } \\
\text { en el momento las condiciones. }\end{array}$
\end{tabular}

Fuente. Elaborado por los autores

\section{Monitoreo, adherencia}

Luego de la implementación de las guías EBE en los servicios piloto, se estableció el proceso de monitoreo y evaluación en la plataforma NQuIRE desde el 2013, para lo cual se identificaron las fuentes de donde se podían extraer los indicadores para alimentar la plataforma, de la siguiente manera:

1. Seguimiento al cronograma de actividades: mediante indicadores, se estableció el cumplimiento de las actividades planificadas.

2. Evaluación del conocimiento previo y posterior a la capacitación en el personal: se elaboraron cuestionarios para medir el conocimiento del personal en el tema. Posteriormente, se realizaron capacitaciones de las guías, y finalmente, fueron aplicados de nuevo cuestionarios al personal.
3. Revisión de indicador de cobertura de capacitación del personal: este indicador resultaba importante por la rotación de personal en la institución.

4. Evaluación del uso de herramientas de valoración en el sistema: por medio de las listas de verificación del registro en el sistema de las escalas valorativas según cada guía.

5. Análisis de los resultados: esto permitió reconocer la forma como las estrategias utilizadas habían sido adoptadas por el personal en las diferentes etapas del proceso y su grado de adaptación.

\section{Proceso desarrollado por etapas}

La medición de los indicadores propuestos en la planeación, permitió conocer el grado deimplementación de la EBE y las debilidades del proceso en cada una de las etapas. 
La adherencia de los profesionales a las recomendaciones implementadas permitió conocer el nivel de cambio alcanzado en la práctica clínica y la efectividad de los resultados obtenidos por la organización en el proceso de cuidado. Estas mediciones se realizaron evaluando diferentes aspectos tales como el nivel de compromiso de la institución, el conocimiento del personal, la aplicación de las recomendaciones y los resultados obtenidos en el proceso de cambio.

Para el logro de lo anterior, se identificaron los aspectos a evaluar por medio de medidas de desempeño con indicadores que permitieron el monitoreo del avance continuo en la adopción de la organización, el personal y los usuarios a través de los resultados obtenidos en cada una de las etapas del proceso de implementación. Es decir, se contaba permanentemente con indicadores de estructura, proceso y resultado.

Esta medición fue planeada desde el inicio del proceso y contó con: un profesional entrenado en la medición de indicadores, diferentes herramientas de medición que describían los pasos y las recomendaciones seleccionadas, lo cual permitió realizar la comparación del avance, y el análisis de los resultados obtenidos. El análisis de resultados permitía la toma de decisiones frente al proceso de monitoreo, con el fin de retroalimentar y definir oportunamente las acciones de mejora en la implementación de las Guías de Práctica Clínica (GPC).

Para proponer estrategias, mecanismos y sistemas de medición y evaluación de la adherencia a las recomendaciones de las GPC, se contempló un marco conceptual que permitiera situar el proceso de adherencia en tres aspectos: en las actividades de adopción e implementación de las GPC en el contexto local, en las acciones de los actores principales de una institución prestadora de servicios de salud y en las dinámicas de transferencia de conocimiento.

Se elaboraron listas de verificación, teniendo en cuenta las recomendaciones de las guías de EBE, con ítems formulados de manera precisa y medible para el proceso de verificación del uso de estas.

Se definió el indicador de adherencia del personal a las recomendaciones para realizar el monitoreo del uso de las recomendaciones de las guías en las diferentes etapas y servicios según cronograma y el desarrollo de la transversalización en la institución.

\section{Porcentaje de adherencia}

Las categorías para el cumplimiento de la meta para el indicador porcentaje de adherencia (tabla 2) se establecieron de la siguiente forma:

Tabla 2. Clasificación de la meta de procedimientos de enfermería FOSCAL

\begin{tabular}{|c|c|}
\hline Categoría & Clasificación \\
\hline Excelente & Mayor o igual al $85 \%$ \\
\hline Bueno & Entre $84.9 \%$ a $76.5 \%$ \\
\hline Por mejorar & Entre $76.4 \%$ a $59.5 \%$ \\
\hline Crítico & Menor a $59.4 \%$ \\
\hline
\end{tabular}

Numero de criterios cumplidos que miden la adherencia a los procedimientos de enfermería *100 Total, criterios evaluados que miden la adherencia a los procedimientos de enfermería.

Fuente. Elaborado por los autores

Al inicio de cada año, durante el periodo 2015 al 2018, se diseñó un cronograma de medición por guía en tiempo y lugar (servicios a evaluar).

Al realizar análisis de las listas de verificación de adherencia para la guía de valoración y manejo del dolor, se hallaron resultados positivos en los períodos evaluados, con una adherencia progresiva, hasta un valor máximo alcanzado de $89 \%$ al final del cuarto año de medición, con lo cual se logró llegar a la meta esperada.

Al realizarse un análisis detallado de los ítems evaluados, se encontró que los que presentaron menor adherencia a las recomendaciones fueron:

- Realizar valoración de dolor aplicando la escala numérica y Campbell según el usuario.

- Realizar y registrar las intervenciones no farmacológicas implementadas para el manejo del dolor.

- Realizar re-valoración periódica del dolor de acuerdo a las intervenciones instauradas.

Para la guía de prevención de caídas y lesiones derivadas de las caídas, la adherencia durante el periodo evaluado mantuvo una tendencia que osciló entre $90.3 \%$ y 94.5\%. Para esta guía, el ítem con menos adherencia fue: la revaluación del estado del usuario a las 24 o 72 
horas, según el procedimiento, utilizando el formato establecido.

Es de resaltar que la guía de prevención de caídas y lesiones derivadas de las caídas fue la primera en transversalizarse en toda la institución, por ser las caídas el evento que más afecta el proceso de atención y los resultados de cuidado. Así, la tasa de caídas para el año 2013 cuando se inició el proceso de implementación de guías fue de 0.91; posteriormente, en el año 2015, cuando se transversalizó institucionalmente, disminuyó a 0.74 y continuó con este comportamiento de tendencia a la baja con el 0.49 hasta el 2017. Para el año 2018, se incluyeron los eventos de caídas ambulatorios, lo que ocasionó un ligero aumento del comportamiento de la tendencia a una tasa de 0.66 .

Existen situaciones que retardan la implementación de las estrategias en el proceso de atención, sobre todo cuando se trata de un cambio o innovación de las recomendaciones implementadas. En el caso de la guía de valoración y manejo del dolor, por no formar parte de la cultura de la institución, se encuentran las siguientes: la valoración, revaloración, intervenciones por enfermería con acciones tendientes a disminuir el dolor, realizar los registros y la satisfacción del usuario o el impacto de las acciones desde la perspectiva del usuario.

Las recomendaciones de las guías de prevención de caídas y lesiones de piel han sido incorporadas al rol o las funciones del personal, transformando la cultura de trabajo y manteniendo una tendencia estable hacia la mejora, en los años del periodo de evaluación y en los diferentes servicios de la institución, lo que ha impactado consecuentemente el proceso de calidad y cuidado a los usuarios.

\section{Sostenibilidad}

Entendida como un proceso continuo, es el cambio en la práctica clínica que se presenta cuando una innovación recién implementada continúa suministrando los beneficios alcanzados en un largo periodo de tiempo. La práctica no retorna al proceso habitual, inclusive después de que el proyecto de implementación ya se haya concretado en su totalidad (15).

La permanencia en el tiempo y las tendencias de mejora, permitieron evidenciar la sostenibilidad de los procesos de EBE implementados, hasta llegar al punto de establecerse como referente en el contexto de atención en enfermería en la región, lo que refleja la incorporación a la cultura del trabajo de la institución. La cultura del trabajo la constituyen las formas de pensar, hacer y transmitir la experiencia vital del trabajo cuando estos procesos devienen, tras una compleja integración, de un componente identitario de naturaleza laboral.

Es importante afianzar el cambio real y mantener una cultura de innovación y evaluación de los resultados obtenidos con el proceso, para lo cual cada año se plantean actividades como:

* Continuar con la medición de indicadores y la socialización de los resultados en los equipos de trabajo.

* Concursos de conocimiento en los diferentes servicios.

* Formación y capacitación anual de nuevos líderes champions.

* Programas de actividades de refuerzo para los líderes.

* Auditoría de la RNAO a la institución, para verificar los avances y sostenibilidad.

* Actualización de las guías y divulgación de las mismas al personal de los servicios.

* Actualización de material educativo utilizado al interior de la institución, tanto para el personal de enfermería, como para los pacientes.

* Talleres de apoyo de acuerdo a cada guía, para el personal involucrado en la atención del paciente o todo el personal involucrado en los procesos relacionados con las mismas.

* Conversatorios con el personal y las líderes de cada guía para analizar aspectos relacionados con la dinámica, el avance alcanzado y las experiencias vividas.

\section{Conclusiones}

La reflexión sobre la práctica de enfermería en las instituciones permite conocer la calidad de los cuidados brindados, identificando los problemas que afectan la práctica y los resultados obtenidos, para así tomar las decisiones que permitan el cambio y la incorporación de modelos de cuidado que impacten esta problemática. 
La metodología de implementación de la RNAO, así como la evaluación y seguimiento de los resultados, evidencia que es posible llevar los resultados de la investigación a la práctica clínica, mejorando la calidad de los cuidados prestados, disminuyendo la variabilidad del cuidado y fortaleciendo el trabajo en equipo.

La adherencia al proceso de implementación de guías de enfermería basada en la evidencia, debe ser medida en las diferentes etapas y a los diferentes participantes, a través de indicadores que permitan visualizar el cambio y sean fuente para la toma de decisiones para la continuidad y éxito de las mismas. De modo que la auditoría y verificación periódica de las estrategias implementadas promueve la adherencia del personal de los servicios en el cumplimiento de las metas institucionales y la sostenibilidad del cambio logrado.

"La transformación de enfermería a través del conocimiento" es el lema propuesto por la RNAO. Es una realidad al evaluar los resultados de cuidado alcanzados después del proceso de implementación, el monitoreo oportuno de los indicadores y el análisis de la tendencia para la toma de decisiones, que permite la sostenibilidad del programa en el tiempo y el avance en nuevas guías a implementar.

Finalmente, se reafirma que la implementación de guías basadas en la evidencia genera un efecto positivo en la calidad de los cuidados brindados, logrando resultados que impactan en la salud y bienestar de los usuarios.

\section{Conflictos de interés}

Los autores declaran no tener conflictos de interés.

\section{Referencias}

1. Sackett DL, Rosenberg WM, Gray JA, Haynes RB, Richardson WS. Evidence Based Medicine: What it is and what it isn't. BMJ. 1996;312(7023):71-72. doi: https://doi.org/10.1136/bmj.312.7023.71

2. Gálvez Toro A, Román Cereto M, Ruiz Román MJ, Hederle Valero C, Morales Asencio JM, Gonzalo E, Romero MN. Enfermería Basada en Evidencias: aportaciones y propuestas. En: I Reunión sobre Enfermería Basada en la Evidencia "Situación actual y propuestas de futuro", convocada por la Fundación Index de Enfermería: 29 de noviembre de 2002, Granada [Internet].Index de Enfermería, 2003 [Citado 22 de agosto de 2019]. 40-41: p. 47-50. Recuperado a partir de: http://www.index-f.com/index-enfermeria/40-41revista/40-41 articulo 47-50.php
3. Avorn, J. Transforming trial results into practice change: The final traslational hurdle: Comment on "Impac of the allhot/jnc7 dissemination project on thiazide-type diurectic use". Arch Intern Med. 2010;170(10):858-860. doi: https://doi.org/10.1001/ archinternmed.2010.125

4. Urra E, Retamal C, Tapias C, Rodríguez M. Enfermería Basada en la Evidencia: Qué es, sus características y dilemas. Invest. educ. enferm. [Internet]. 2010 [Citado 22 de agosto de 2019];28(1):108-118. Recuperado a partir de: http://www.scielo.org.co/scielo.php?script=sci abstract\&pid $=$ S0120-53072010000100013

5. Grinspun D, Bajnok I. Transforming Nursing Through Knowledge. Chapter 12, RNAO's global spread of BPGS: The BPSO designation sustainability and fidelity. [Internet]. Indianapolis: Sigma Theta Tau International. 2018 [citado 22 agosto de 2019] 288-313p. Recuperado a partir de: https://sigma.nursingrepository.org/bitstream/ handle/10755/624122/FreeChapter_Grinspun. pdf? sequence $=8 \&$ isAllowed $=y$

6. Ministerio de Salud Subsecretaría de redes asistenciales. Norma General Administrativa No. 19, "Gestión del Cuidado de Enfermería para la atención Cerrada". [Internet]. Chile; 2007 [Citado 22 agosto de 2019]. Recuperado a partir de: https://enfermeriachl. files.wordpress.com/2018/11/resolucion-nc2b01127-norma-nc2b0-19.pdf

7. Ministerio de Salud y Protección Social, Instituto de Evaluación Tecnológica en Salud. Manual Metodológico para la Medición y Evaluación de la Adherencia Sostenible a las Recomendaciones de las Guías de Práctica Clínica [Internet] Bogotá; 2017 [Citado 22 agosto de 2019]. Recuperado a partir de: https://docplayer.es/113515126-Manualmetodologico-para-la-medicion-y-evaluacion-dela-adherencia-sostenible-a-las-recomendaciones-delas-guias-de-practica-clinica.html

8. Registered Nurses' Association of Ontario. Guía de Buenas Prácticas. Herramienta de Implantación de Buenas Prácticas. 2nd ed. [Internet]. 2012 [citado 22 de agosto de 2019] Recuperado a partir de: https:// rnao.ca/sites/rnao-ca/files/Toolkit en15.pdf

9. Registered Nurses' Association of Ontario. Best Practice Guidelines. [Internet]. 2013. [citado 22 de agosto de 2019] Recuperado a partir de: http://rnao. ca/bpg/guidelines/clinical

10. Grinspun D. Bajnok I. Transforming Nursing Through Knowledge. Chapter 12, RNAO's global spread of BPGS: The BPSO designation sustainability and fidelity. [Internet]. Indianapolis: Sigma Theta Tau International. 2018 [Citado 22 de agosto de 2019] Recuperado a partir de: 
https://sigma.nursingrepository.org/bitstream/ handle/10755/624122/FreeChapter_Grinspun. pdf? sequence $=8 \&$ isAllowed $=y$

11. Registered Nurses' Association of Ontario. Best Practice Spotlight Organization. Noviembre de 2013. Disponible en https://rnao.ca/bpg/bpso

12. Evidence-based health care. An open learning resources for health care professionals. Unit 4: Acting on the evidence. Critical Skills Appraisal Programme and Health Care Oxford: Libraries Unit. Luton: Chiltem Press; 1999.

13. Cañón A., Hilda María, La práctica de la enfermería basada en la evidencia. Investigación en Enfermería: Imagen y Desarrollo [Internet]. 2007;9(2):101110. [Citado 22 agosto de 2019] Recuperado a partir de: https://www.redalyc.org/articulo. oa? id $=145212857002$.

14. Subirana, Mireia. EBE. Recurso para unos cuidados seguros. Unidad 1.[Internet] Difusión Avances de Enfermería (DAE); 2010. [Citado 22 agosto de 2019] Recuperado a partir de: https://www.auladae. com/pdf/cursos/capitulo/ebe.pdf.

15. Ministerio de Salud y Protección Social, Instituto de Evaluación Tecnológica en Salud. Manual Metodológico para la Medición y Evaluación de la Adherencia Sostenible a las Recomendaciones de las Guías de Práctica Clínica [Internet] Bogotá; 2017 [Citado 22 agosto de 2019]. Recuperado a partir de: https://docplayer.es/113515126-Manualmetodologico-para-la-medicion-y-evaluacion-dela-adherencia-sostenible-a-las-recomendaciones-delas-guias-de-practica-clinica.html 